\title{
Nonlocal Problems Arising in Thermoelectrics
}

\author{
Jaywan Chung' and Byungki Ryu' ${ }^{2}$ \\ ${ }^{1}$ Department of Mathematics, Dankook University, Cheonan-si 330-714, Republic of Korea \\ ${ }^{2}$ Thermoelectric Conversion Research Center, Korea Electrotechnology Research Institute (KERI), \\ Changwon-si 642-120, Republic of Korea
}

Correspondence should be addressed to Jaywan Chung; jaywan.chung@gmail.com

Received 30 October 2014; Accepted 2 December 2014; Published 15 December 2014

Academic Editor: Yuji Liu

Copyright (C) 2014 J. Chung and B. Ryu. This is an open access article distributed under the Creative Commons Attribution License, which permits unrestricted use, distribution, and reproduction in any medium, provided the original work is properly cited.

We revisit the derivation of a nonlocal problem modelling temperature distribution due to power generation using thermoelectric effect. The problem has nonlocal coefficients in reaction and convection terms rather than diffusion term, which makes the problem more interesting. In this paper, we prove that the nonlocal problem has a unique decreasing solution when electric current induced by the thermoelectric effect is small enough.

\section{Introduction}

Consider a boundary value problem

$$
\begin{aligned}
& y^{\prime \prime}+\lambda^{2} \rho(y)-\lambda \frac{d \alpha}{d y} y y^{\prime}=0, \quad \text { for } 0 \leq x \leq l, \\
& y(0)=y_{H}, \quad y(l)=y_{C}, \quad y_{H}>y_{C}>0 .
\end{aligned}
$$

Here $y=y(x), \alpha=\alpha(y)$, and the prime' indicates derivative with respect to $x$. When $\lambda$ is constant, (1) is a usual nonlinear convection-reaction-diffusion equation. However, in this paper, $\lambda$ is a nonlocal coefficient given by

$$
\lambda=\frac{\int_{y_{C}}^{y_{H}} \alpha(y) d y}{\left(\int_{0}^{l} \rho(y(x)) d x+\gamma\right)}
$$

for some positive constant $\gamma$. Note that $\lambda$ is nonlocal due to the integral $\int_{0}^{l} \rho(y(x)) d x$ which depends on the solution $y$.

There are extensive literatures on nonlocal problems, but the nonlocality can be different for each problem. Usually it refers to nonlocal diffusion such as the fractional Laplacian (see, e.g., [1-5]), but some problems contain a nonlocal diffusivity, which is in the coefficient of the usual Laplacian term [6-9]. Also there is a problem having a nonlocal boundary condition [10]. However our problem (1)-(2) has a nonlocal convection-reaction term, which is another type of nonlocality. Even more interestingly, the presence of $\lambda^{2}$ indicates that nonlocality is nonlinear.

Problem (1)-(2) models temperature distribution under thermoelectric effect, which refers to direct conversion between electricity and thermal energy [11-14]. When there is temperature difference, it induces voltage and electric current because charge carriers move and diffuse from hot side to cold side (which is called Seebeck effect). On the other hand, the charge carriers also carry kinetic energy so that there is a flow of kinetic energy or thermal current when there is electric current (which is called Peltier effect).

The thermoelectric effect is applicable to electric power generation and solid state cooling [12, 14]. For example, with an advantage of small size and long time stability in extreme condition, it has been applied for power generation in spacecraft, as a form of a radioisotope thermoelectric generator (RTG). Also it can increase the system efficiency of vehicles by harvesting the waste heat. Our problem (1)(2) models the power generation; the temperature difference between boundary points induces electric current, and the heat generated by the electric current alters the temperature distribution. The nonlocal coefficient $\lambda$ in (2) represents the induced electric current.

For the application of thermoelectric technology, understanding of thermal properties is essential. There have been 
many studies using numerical (see, e.g., $[15,16])$ and analytical $[12,13,17,18]$ approaches to calculate the temperature distribution and energy conversion efficiency or to find new thermoelectric parameters. However, to the best of our knowledge, few have paid attention to the mathematical analysis of the nonlocal PDE (1)-(2).

In this paper, we revisit the modelling of (1)-(2) and prove its well-posedness for some particular cases. Furthermore, we prove that the solution has no interior maximum; that is, the temperature distribution is monotonic when the electric current density is small enough. For a wide class of PDEs along the heat equation, the monotonicity of solutions (which is physically obvious) can be explained by the maximum principle. But in our problem, the maximum principle does not hold and, surprisingly, our result shows that there can be an interior maximum temperature when the physical property of thermoelectric material is very good; for example, the thermoelectric figure of merit is very large.

\section{Modelling}

The thermoelectric effect has been well known among physicists [11]. But the derivation of problem (1)-(2) is not easily accessible in the literature, so we revisit the derivation in this section.

2.1. Derivation in Physical Quantities. The electrical current density $\mathbf{J}$ and the thermal current density $\mathbf{J}^{\mathrm{Q}}$ are generated if there is electromotive force such as electric field $\boldsymbol{\epsilon}$ or temperature gradient $\nabla T$. More precisely, their quantitative relations are

$$
\begin{aligned}
\mathbf{J} & =\sigma(\boldsymbol{\epsilon}-\alpha \nabla T), \\
\mathbf{J}^{\mathrm{Q}} & =\Pi \mathbf{J}-\kappa \nabla T,
\end{aligned}
$$

where $\sigma, \alpha, \Pi, \kappa$, and $T$ are electric conductivity, Seebeck coefficient, Peltier coefficient, thermal conductivity, and the absolute temperature, respectively. Here $\sigma, \alpha, \Pi$, and $\kappa$ can be 3 by 3 tensors, but to simplify model we assume that they are scalar quantities. We also assume that they depend on the temperature only:

$$
\begin{aligned}
\sigma & =\sigma(T), \\
\alpha & =\alpha(T), \\
\Pi & =T \alpha(T), \\
\kappa & =\kappa(T),
\end{aligned}
$$

where the third relation, $\Pi=T \alpha$, can be justified by Onsager's relation $[11,19]$. If the electrical current density is nonvanishing, there will be an electrical power dissipation $\boldsymbol{\epsilon} \cdot \mathbf{J}$, acting as a heat source. Hence by the energy conservation law, local energy density $u$ satisfies

$$
\frac{\partial u}{\partial t}+\nabla \cdot \mathbf{J}^{Q}=\boldsymbol{\epsilon} \cdot \mathbf{J}
$$

Furthermore, the time derivative of the local energy density is written as $\partial u / \partial t=C(\partial T / \partial t)$, where $C$ is the heat capacity of a thermoelectric material and $T$ is the temperature therein.

We have derived so far

$$
\begin{aligned}
C \frac{\partial T}{\partial t} & =-\nabla \cdot \mathbf{J}^{Q}+\boldsymbol{\epsilon} \cdot \mathbf{J}, \\
\boldsymbol{\epsilon} & =\rho \mathbf{J}+\alpha \nabla T, \\
\mathbf{J}^{Q} & =\alpha \mathbf{J}-\kappa \nabla T,
\end{aligned}
$$

where $\rho=1 / \sigma$ is resistivity. Hence, combining them,

$$
\begin{aligned}
C \frac{\partial T}{\partial t}= & -\nabla \cdot(\alpha T \mathbf{J}-\kappa \nabla T)+(\rho \mathbf{J}+\alpha \nabla T) \cdot \mathbf{J} \\
= & \rho|\mathbf{J}|^{2}-T \frac{d \alpha}{d T}(\nabla T \cdot \mathbf{J})-\alpha T(\nabla \cdot \mathbf{J}) \\
& +\nabla \cdot(\kappa \nabla T) .
\end{aligned}
$$

Because charge carrier is not generated in the material, $\nabla \cdot \mathbf{J}=$ 0 and

$$
C \frac{\partial T}{\partial t}=\rho|\mathbf{J}|^{2}-T \frac{d \alpha}{d T}(\nabla T \cdot \mathbf{J})+\nabla \cdot(\kappa \nabla T) .
$$

Now we need boundary conditions. Here we consider power generation by a thermoelectric material, which is operated by imposing hot-side temperature $T_{H}$ and cold-side temperature $T_{C}$ at the boundary:

$$
\begin{aligned}
& T(x=0)=T_{H}, \\
& T(x=L)=T_{C},
\end{aligned}
$$

where $L$ is the length of the thermoelectric material.

Finally, to determine the electric current density J, we integrate the second equation in (6) to obtain

$$
\int \rho \mathbf{J} \cdot d \mathbf{r}=\int(\boldsymbol{\epsilon}-\alpha \nabla T) \cdot d \mathbf{r}
$$

Since $\mathbf{J}=J$ is assumed to be a constant scalar, the left-hand side is $J \int \rho d x$ and the right-hand side is $-\Delta \psi-\int \alpha d T$, where $\Delta \psi$ is a voltage drop. When the resistance outside the material is fixed as $R_{\text {ext }}$, the voltage drop is equal to $J A R_{\text {ext }}$. Therefore we have

$$
J \int \rho d x=-J A R_{\mathrm{ext}}-\int \alpha d T .
$$

Note that $\int \alpha d T$ is a constant. 
2.2. Nondimensionalization. Assuming that $C$ and $\kappa$ are constant, we nondimensionalize the equation by introducing the following dimensionless parameters:

$$
\begin{aligned}
y & =\frac{T}{\Delta T}, \\
\bar{x} & =\frac{x}{A / L}, \\
\bar{t} & =\frac{\kappa}{C(A / L)^{2}} t, \\
\bar{\rho} & =\frac{\rho}{R_{\mathrm{ref}} A / L}, \\
\lambda & =\sqrt{\frac{(A / L)^{3} R_{\mathrm{ref}}}{\kappa \Delta T}} J, \\
\bar{\alpha} & =\sqrt{\frac{\Delta T}{\kappa R_{\mathrm{ref}} A / L}} \alpha,
\end{aligned}
$$

where $\Delta T=T_{H}-T_{C}$ and $R_{\text {ref }}$ is a reference resistance. Then the first equation in (6) becomes

$$
\frac{\partial y}{\partial \bar{t}}=\lambda^{2} \bar{\rho}-\lambda \frac{d \bar{\alpha}}{d y} y \frac{\partial y}{\partial \bar{x}}+\frac{\partial^{2} y}{\partial \bar{x}^{2}} .
$$

Also the nonlocal equation (11) becomes

$$
\lambda \int_{0}^{L^{2} / A} \bar{\rho} d \bar{x}=-\gamma \lambda-\int_{T_{H} / \Delta T}^{T_{C} / \Delta T} \bar{\alpha} d y
$$

where $\gamma=\left(L^{2} / A\right)\left(R_{\text {ext }} / R_{\text {ref }}\right)$. And the boundary condition (9) becomes

$$
\begin{array}{r}
y(\bar{x}=0)=\frac{T_{H}}{\Delta T}, \\
y\left(\bar{x}=\frac{L^{2}}{A}\right)=\frac{T_{C}}{\Delta T} .
\end{array}
$$

In summary, omitting the bars in $\bar{x}, \bar{t}, \bar{\rho}$, and $\bar{\alpha}$, we have a nonlocal initial-boundary value problem:

$$
\begin{aligned}
& y_{t}=y_{x x}+\lambda^{2} \rho(y)-\lambda \frac{d \alpha}{d y} y y_{x}, \\
& \lambda=\frac{\int_{y_{C}}^{y_{H}} \alpha(y) d y}{\left(\int_{0}^{l} \rho(y(x)) d x+\gamma\right)}, \\
& y(0, t)=y_{H}, \quad y(l, t)=y_{C}, \quad \forall t>0, \\
& y(x, 0)=y_{0}(x), \quad \text { for } 0 \leq x \leq l,
\end{aligned}
$$

where $l:=L^{2} / A, y_{H}:=T_{H} / \Delta T$, and $y_{C}:=T_{C} / \Delta T$.

\section{Well-Posedness of Stationary Problems}

When there is no temporal change in the solution, (16) reduces to the following nonlocal boundary value problem:

$$
\begin{aligned}
& y^{\prime \prime}+\lambda^{2} \rho(y)-\lambda \frac{d \alpha}{d y} y y^{\prime}=0, \\
& \lambda=\frac{\int_{y_{C}}^{y_{H}} \alpha(y) d y}{\left(\int_{0}^{l} \rho(y(x)) d x+\gamma\right)}, \\
& y(0)=y_{H}, \quad y(l)=y_{C}, \quad y_{H}>y_{C}>0 .
\end{aligned}
$$

Since the resistivity $\rho$ is positive, the reaction term $\lambda^{2} \rho$, which corresponds to the Joule heating, is also positive, so there is a heat source in the domain. Our thermoelectric power generation system is limited to the case when $\gamma>0$, meaning that there is no power source other than the thermoelectric material. Note that $\lambda$ and $\alpha$ have the same sign. Physically, there are two types of thermoelectric materials: one is p-type thermoelectric material with positive charge hole carrier $(\alpha>0)$ and the other is n-type thermoelectric material with negative charge electron carrier $(\alpha<0)$. To simplify arguments, we only consider the p-type case where $\alpha>0$; thereby the current flows from hot side to cold side $(\lambda>0)$.

Most thermoelectric materials are degenerated semiconductors and therefore their resistivities are increasing with respect to the temperature. But the Seebeck coefficient $\alpha$ can be concave if there is bipolar effect. To simplify the situation, we assume that there is no bipolar effect and the resistivity $\rho$ and the Seebeck coefficient $\alpha$ are constant or linear.

In this section, for those particular cases, we will prove the well-posedness of the nonlocal problem (17) and give some sufficient smallness conditions on $\lambda$ to guarantee that the solution is decreasing in space.

3.1. When $\rho$ and $\alpha$ Are Constants. If we assume that $\rho(y)=\rho_{0}$ and $\alpha(y)=\alpha_{0}$, the nonlocal boundary value problem (17) becomes local and linear:

$$
\begin{aligned}
& y^{\prime \prime}+\lambda^{2} \rho_{0}=0, \quad \lambda=\alpha_{0} \frac{y_{H}-y_{C}}{\rho_{0} l+\gamma}, \\
& y(0)=y_{H}, \quad y(l)=y_{C}, \quad y_{H}>y_{C}>0 .
\end{aligned}
$$

If we let $y_{\Delta}:=y_{H}-y_{C}$, an exact solution is

$$
y(x)=-\frac{\lambda^{2} \rho_{0}}{2} x^{2}+\left(\frac{\lambda^{2} \rho_{0} l}{2}-\frac{y_{\Delta}}{l}\right) x+y_{H} .
$$

Note that $y_{\Delta}$ is equal to 1 if we consider the dimensional quantities. But in order not to lose mathematical generality, we assume that $y_{\Delta}$ can be arbitrarily positive. It is easy to verify that there is no interior maximum if and only if

$$
\lambda^{2} \rho_{0} l^{2} \leq 2 y_{\Delta}
$$

To understand the physical meaning of (20), we consider the corresponding physical quantities and obtain a condition 
between material property and thermoelectric power generation resistance:

$$
\frac{\alpha^{2}}{\rho \kappa}<\frac{2(1+\Gamma)^{2}}{\left(T_{H}-T_{C}\right)}
$$

where $\Gamma$ is the ratio of the external to the internal resistance. Note that the left-hand side is a pure material parameter determined by $\alpha, \rho$, and $\kappa$, but the right-hand side is a pure device parameter determined by $\Gamma$ and temperature difference. Also note that the value in the left-hand side is the definition of $Z$ value in thermoelectrics. The thermoelectric figure of merit $Z T_{m}$, where $T_{m}=\left(T_{H}+T_{C}\right) / 2$, estimates the system efficiency: large $Z T_{m}$ means high efficiency [13]. Now consider a thermoelectric system operating between $T_{H}=500 \mathrm{~K}$ and $T_{C}=300 \mathrm{~K}$, assuming that the system is designed to have maximum output power; that is, $\Gamma=1$. Then there is no interior maximum if and only if $Z T_{m}$ is less than 16 . Hence it is difficult to observe interior maximum in common thermoelectric power generation systems because the world highest thermoelectric figure of merit $Z T$ is 2.6 [20]. However, it is remarkable that there can be interior maximum if a better thermoelectric material with high $Z T$ value is developed. Since the thermoelectric properties are slowly varying function of $T$, the physical meaning of (21) will be almost similar for real world.

3.2. When $\rho$ Is Constant and $\alpha$ Is Linear. Assume that $\rho(y)=$ $\rho_{0}$ and $\alpha(y)=\alpha_{0}+\alpha_{1} y$ where $\rho_{0}, \alpha_{0} \geq 0$ and $\alpha_{1}>0$. Then the nonlocal problem (17) becomes local but nonlinear:

$$
\begin{aligned}
& y^{\prime \prime}+\lambda^{2} \rho_{0}-\lambda \alpha_{1} y y^{\prime}=0, \\
& \lambda=\frac{y_{\Delta}}{\left(\rho_{0} l+\gamma\right)}\left(\alpha_{0}+\frac{\alpha_{1}}{2}\left(y_{H}+y_{C}\right)\right), \\
& y(0)=y_{H}, \quad y(L)=y_{C}, \quad y_{H}>y_{C}>0 .
\end{aligned}
$$

To show that there is a unique solution under smallness condition, we use a classical well-posedness result [21, Theorem 3.7].

Theorem 1 (well-posedness of a nonlinear boundary value problem). Consider a nonlinear boundary value problem

$$
\begin{aligned}
& y^{\prime \prime}(x)+f\left(x, y(x), y^{\prime}(x)\right)=0, \\
& y(0)=y(l)=0 .
\end{aligned}
$$

Suppose that $f\left(x, y, y^{\prime}\right)$ are continuous on

$$
[0, l] \times\left[-M_{y}, M_{y}\right] \times\left[\frac{-4 M_{y}}{l}, \frac{4 M_{y}}{l}\right]
$$

and satisfy a Lipschitz condition

$$
\begin{aligned}
& \left|f\left(x, y_{1}, y_{1}^{\prime}\right)-f\left(x, y_{2}, y_{2}^{\prime}\right)\right| \\
& \quad \leq K_{1}\left|y_{1}-y_{2}\right|+K_{2}\left|y_{1}^{\prime}-y_{2}^{\prime}\right|
\end{aligned}
$$

there. Let

$$
\begin{gathered}
M_{0}:=\max _{0 \leq x \leq l}|f(x, 0,0)|, \\
M_{f}:=\max \left|f\left(x, y, y^{\prime}\right)\right|, \\
\text { for }|y| \leq M_{y}, \quad\left|y^{\prime}\right| \leq \frac{4 M_{y}}{l}, \quad x \in[0, l] .
\end{gathered}
$$

Then if

$$
\beta:=\frac{K_{1} l^{2}}{8}+\frac{K_{2} l}{2}<1
$$

and either

$$
\frac{M_{0} l^{2}}{8} \leq M_{y}(1-\beta) \quad \text { or } \quad \frac{M_{f} l^{2}}{8} \leq M_{y},
$$

then problem (23) has one and only one solution $y(x)$ such that

$$
\begin{array}{r}
|y(x)| \leq M_{y}, \\
\left|y^{\prime}(x)\right| \leq \frac{4 M_{y}}{l}, \\
\text { for } x \in[0, l] .
\end{array}
$$

The proof is based on the contraction mapping principle applied to the Green function representation of solutions. Using this theorem, we can show that there is a unique solution of (22).

Proposition 2 (existence of a decreasing solution). Suppose that

$$
\begin{aligned}
& \frac{1}{2}|\lambda| \alpha_{1} l\left(\frac{3}{4} y_{\Delta}+y_{H}\right)<1, \\
& \lambda^{2} \rho_{0} l^{2}+\frac{1}{2}|\lambda| \alpha_{1} l y_{\Delta}\left(y_{\Delta}+4 y_{H}\right) \leq 2 y_{\Delta} .
\end{aligned}
$$

Then there is a unique solution of (22) such that

$$
\begin{array}{r}
|y(x)-s(x)| \leq \frac{y_{\Delta}}{4}, \\
\left|y^{\prime}(x)-s^{\prime}(x)\right| \leq \frac{y_{\Delta}}{l}, \\
\text { for } x \in[0, l],
\end{array}
$$

where $s(x):=\left(y_{H}(l-x)+y_{C} x\right) / l$. Furthermore, the solution is decreasing in $x$ (hence there is no interior maximum).

Proof. Let $w$ be a solution of (22). Then

$$
y(x):=w(x)-s(x)
$$

satisfies (23) with $f\left(x, y, y^{\prime}\right):=\lambda^{2} \rho_{0}-\lambda \alpha_{1}(y+s)\left(y^{\prime}+s^{\prime}\right)$. Suppose that $|y| \leq M_{y}$ and $\left|y^{\prime}\right| \leq 4 M_{y} / l$. Then we can easily verify that the Lipschitz condition (25) holds for $K_{1}=$ $|\lambda| \alpha_{1}\left(4 M_{y}+y_{\Delta}\right) / l$ and $K_{2}=|\lambda| \alpha_{1}\left(M_{y}+y_{H}\right)$. Hence

$$
\begin{aligned}
\beta & =\frac{1}{2}|\lambda| \alpha_{1} l\left(2 M_{y}+\frac{y_{\Delta}}{4}+y_{H}\right), \\
M_{f} & =\lambda^{2} \rho_{0}+|\lambda| \alpha_{1}\left(M_{y}+y_{H}\right)\left(\frac{4 M_{y}+y_{\Delta}}{l}\right) .
\end{aligned}
$$


If we choose $M_{y}:=y_{\Delta} / 4$, then, by the assumption, $\beta<1$ and $M_{f} l^{2} / 8 \leq M_{y}$. Hence the first conclusion follows from Theorem 1 .

From the conclusion on the bound of $y^{\prime}$ in Theorem 1,

$$
\frac{y_{\Delta}}{l} \geq\left|y^{\prime}(x)\right|=\left|w^{\prime}(x)-s^{\prime}(x)\right|=\left|w^{\prime}(x)+\frac{y_{\Delta}}{l}\right| \text {. }
$$

Therefore $w^{\prime}(x) \leq 0$ and the solution is decreasing.

Note that when $\alpha_{1}=0$, the conditions in Proposition 2 are reduced to the exact condition (20) in the previous section.

3.3. When $\rho$ Is Linear and $\alpha$ Is Constant. If we assume that $\rho(y)=\rho_{1} y$ and $\alpha(y)=\alpha_{0}$, where $\rho_{1}, \alpha_{0} \geq 0$, the nonlocal boundary value problem (17) becomes linear:

$$
\begin{aligned}
& y^{\prime \prime}+\lambda^{2} \rho_{1} y=0, \\
& \lambda\left(\rho_{1} \int_{0}^{l} y(x) d x+\gamma\right)=\alpha_{0} y_{\Delta}, \\
& y(0)=y_{H}, \quad y(l)=y_{C}, \quad y_{H}>y_{C}>0 .
\end{aligned}
$$

With a given nonnegative $\lambda$, the boundary value problem without the nonlocal condition on $\lambda$ has an exact solution if $\mu:=\lambda \sqrt{\rho_{1}}<\pi / l$ :

$$
y(x ; \lambda)=\frac{y_{C}-y_{H} \cos \mu l}{\sin \mu l} \sin \mu x+y_{H} \cos \mu x .
$$

Integrating the solution yields

$$
\begin{aligned}
\int_{0}^{l} y(x ; \lambda) d x= & \frac{y_{C}-y_{H} \cos \mu l}{\mu \sin \mu l}(1-\cos \mu l) \\
& +y_{H} \frac{\sin \mu l}{\mu} .
\end{aligned}
$$

Due to $\sin \mu l$ in the denominator of the first term, the integral diverges to $\infty$ as $\mu \uparrow \pi / l$. Furthermore, by comparison principle, the solution $y(x ; \lambda)$ increases strictly as $\mu$ increases. Therefore, $\int_{0}^{l} y(x ; \lambda) d x$ is strictly increasing to $\infty$ as $\lambda \uparrow$ $\pi /\left(\sqrt{\rho_{1}} l\right)$.

So the left-hand side of the second equation in (35) strictly increases from 0 to $\infty$ as $\lambda$ increases from 0 to $\pi /\left(\sqrt{\rho_{1}} l\right)$. Therefore there is one and only one $\lambda$ satisfying the second equation, which proves the unique existence.

Since $y^{\prime \prime}=-\lambda^{2} \rho_{1} y \leq 0$, the solution is decreasing if and only if $y^{\prime}(0) \leq 0$. From the exact solution, this condition is equivalent to

$$
\frac{y_{C}}{y_{H}} \leq \cos \left(\lambda \sqrt{\rho_{1}} l\right)
$$

We summarize the result.

Proposition 3. There is a solution of (35). If $\lambda^{2} \rho_{1} l^{2}<\pi^{2}$, the solution is unique. Furthermore, if (38) holds, the solution is decreasing in $x$.
3.4. When $\rho$ and $\alpha$ Are Linear. Assume that $\rho(y)=\rho_{1} y$ and $\alpha(y)=\alpha_{0}+\alpha_{1} y$, where $\rho_{1}, \alpha_{0} \geq 0$ and $\alpha_{1}>0$. Then problem (17) becomes

$$
\begin{aligned}
& y^{\prime \prime}+\lambda^{2} \rho_{1} y-\lambda \alpha_{1} y y^{\prime}=0, \\
& \lambda\left(\rho_{1} \int_{0}^{l} y(x) d x+\gamma\right)=y_{\Delta}\left(\alpha_{0}+\frac{\alpha_{1}}{2}\left(y_{H}+y_{C}\right)\right), \\
& y(0)=y_{H}, \quad y(l)=y_{C}, \quad y_{H}>y_{C}>0 .
\end{aligned}
$$

With a given small nonnegative $\lambda$, we can show that there is a unique, decreasing solution of the boundary value problem without the second equation. Then the decrease of the solution guarantees that a $\lambda$ satisfying the second equation is unique.

Theorem 4 (existence of a decreasing solution). Let

$$
\begin{aligned}
\Lambda_{\beta}:=\{\lambda: & \left.\frac{1}{8} \lambda^{2} \rho_{1} l^{2}+\frac{1}{2} \lambda \alpha_{1} l\left(\frac{3}{4} y_{\Delta}+y_{H}\right)<1\right\}, \\
\Lambda_{f}:= & \left\{\lambda: \frac{1}{4} \lambda^{2} \rho_{1} l^{2}\left(y_{\Delta}+4 y_{H}\right)\right. \\
& \left.+\frac{1}{2} \lambda \alpha_{1} l y_{\Delta}\left(y_{\Delta}+4 y_{H}\right) \leq 2 y_{\Delta}\right\},
\end{aligned}
$$

and $\lambda^{*}:=\sup \Lambda_{\beta} \cap \Lambda_{f}$. If

$$
\lambda^{*}\left(\frac{\rho_{1}}{2} y_{\Delta} l+\lambda\right)>y_{\Delta}\left(\alpha_{0}+\frac{\alpha_{1}}{2}\left(y_{H}+y_{C}\right)\right)
$$

there is a unique solution of (39) with a $\lambda<\lambda^{*}$. Furthermore, the solution is decreasing in $x$ and satisfies

$$
\begin{array}{r}
|y(x)-s(x)| \leq \frac{y_{\Delta}}{4}, \\
\left|y^{\prime}(x)-s^{\prime}(x)\right| \leq \frac{y_{\Delta}}{l}, \\
\text { for } x \in[0, l]
\end{array}
$$

where $s(x):=\left(y_{H}(l-x)+y_{C} x\right) / l$.

Proof. First we disregard the second equation in (39) and prove that there is a unique, decreasing solution with a given nonnegative $\lambda<\lambda^{*}$. The proof is almost the same as the one of Proposition 2. Let $w$ be a solution of (39), disregarding the second equation. Then $y(x):=w(x)-s(x)$ satisfies (23) with $f\left(x, y, y^{\prime}\right):=\lambda^{2} \rho_{1}(y+s)-\lambda \alpha_{1}(y+s)\left(y^{\prime}+s^{\prime}\right)$. Suppose that $|y| \leq M_{y}$ and $\left|y^{\prime}\right| \leq 4 M_{y} / l$. Then we can verify that the Lipschitz condition (25) holds for $K_{1}=\lambda^{2} \rho_{1}+\lambda \alpha_{1}\left(4 M_{y}+\right.$ $\left.y_{\Delta}\right) / l$ and $K_{2}=\lambda \alpha_{1}\left(M_{y}+y_{H}\right)$. Hence

$$
\begin{aligned}
\beta= & \frac{1}{8} \lambda^{2} \rho_{1} l^{2}+\frac{1}{2} \lambda \alpha_{1} l\left(2 M_{y}+\frac{1}{4} y_{\Delta}+y_{H}\right), \\
M_{f}= & \lambda^{2} \rho_{1}\left(M_{y}+y_{H}\right) \\
& +\lambda \alpha_{1}\left(M_{y}+y_{H}\right)\left(\frac{4 M_{y}+y_{\Delta}}{l}\right) .
\end{aligned}
$$


If we choose $M_{y}:=y_{\Delta} / 4$, then, because $\lambda \in \Lambda_{\beta} \cap \Lambda_{f}, \beta<$ 1 and $M_{f} l^{2} / 8 \leq M_{y}$. Hence well-posedness follows from Theorem 1. Also from the conclusion on the bound of $y^{\prime}$ in Theorem 1,

$$
\frac{y_{\Delta}}{l} \geq\left|y^{\prime}(x)\right|=\left|w^{\prime}(x)+\frac{y_{\Delta}}{l}\right|
$$

which implies that $w^{\prime}(x) \leq 0$ and the solution is decreasing.

Now it suffices to prove there is a unique $\lambda<\lambda^{*}$ with which the second equation in (39) holds. Because $\int_{0}^{l} y(x) d x \geq$ $(1 / 2) y_{\Delta} l$, as $\lambda \uparrow \lambda^{*}$, the left-hand side of the second equation in (39) is bounded below by $\lambda^{*}\left(\left(\rho_{1} / 2\right) y_{\Delta} l+\gamma\right)$. Hence, by the assumption (41), there is a $\lambda<\lambda^{*}$ satisfying the second equation. Uniqueness of such $\lambda$ can be proven by comparison principle. Let $y_{1}, y_{2}$ be solutions of (39) with $\lambda=\lambda_{1}, \lambda_{2}$, respectively. If $\lambda_{1} \leq \lambda_{2} \leq \lambda^{*}$, the solutions $y_{1}$ and $y_{2}$ are decreasing in $x$. Define $\mathscr{L}[y]:=-y^{\prime \prime}-\lambda_{1}^{2} \rho_{1} y+\lambda_{1} \alpha_{1} y y^{\prime}$. Then $\mathscr{L}\left[y_{1}\right] \equiv 0$ and

$$
\mathscr{L}\left[y_{2}\right]=\left(\lambda_{2}^{2}-\lambda_{1}^{2}\right) \rho_{1} y_{2}-\left(\lambda_{2}-\lambda_{1}\right) \alpha_{1} y_{2} y_{2}^{\prime} \geq 0
$$

Hence $y_{2} \geq y_{1}$. But due to the second equation in (39),

$$
\begin{aligned}
\lambda_{2}\left(\rho_{1} \int_{0}^{l} y_{2}(x) d x+\gamma\right) & =\lambda_{1}\left(\rho_{1} \int_{0}^{l} y_{1}(x) d x+\gamma\right) \\
& \leq \lambda_{1}\left(\rho_{1} \int_{0}^{l} y_{2}(x) d x+\gamma\right)
\end{aligned}
$$

which implies $\lambda_{2} \leq \lambda_{1}$. Therefore $\lambda_{1}=\lambda_{2}$, which proves the uniqueness of $\lambda$.

Remark 5. When $\alpha_{1}=0$, the condition $\lambda \in \Lambda_{\beta}$ in Theorem 4 reduces to $\lambda^{2} \rho_{1} l^{2}<8$, which is a stronger assumption than the condition $\lambda^{2} \rho_{1} l^{2}<\pi^{2}$ in Proposition 3. Also the condition $\lambda \in \Lambda_{f}$ reduces to

$$
\frac{y_{C}+y_{\Delta} / 4}{y_{H}+y_{\Delta} / 4} \leq 1-\frac{1}{2} \lambda^{2} \rho l^{2}
$$

which is a stronger assumption than condition (38) because

$$
\frac{y_{C}}{y_{H}} \leq \frac{y_{C}+y_{\Delta} / 4}{y_{H}+y_{\Delta} / 4} \leq 1-\frac{1}{2} \lambda^{2} \rho l^{2} \leq \cos \left(\lambda \sqrt{\rho_{1}} l\right) \text {. }
$$

Remark 6. In the previous section, we observed that $\int_{0}^{l} y(x ; \lambda) d x$ diverges to $\infty$ as $\lambda$ increases. This observation suggests that the same can happen to (39). If then, condition (41) is not necessary to guarantee the existence of a solution. Hence the divergent behavior of the integral needs further investigation.

\section{Conclusions}

When the resistivity $\rho$ and the Seebeck coefficient $\alpha$ are constant or linear, we have found some sufficient smallness conditions on the electric current $\lambda$ which ensures that the nonlocal problem (17) has a unique decreasing solution. Also if the condition does not hold, a solution can have interior maximum, which is physically surprising. The fact can draw attention of engineers designing a thermoelectric system.

When both $\rho$ and $\alpha$ are linear, we needed an ad hoc condition to guarantee the existence of a solution, but it seems not necessary. Eliminating the ad hoc condition and proving the existence and uniqueness for arbitrary $\lambda$ will be a further interest.

\section{Conflict of Interests}

The authors declare that there is no conflict of interests regarding the publication of this paper.

\section{Acknowledgments}

This work was supported by the Energy Efficiency \& Resource Core Technology Program of the Korea Institute of Energy Technology Evaluation and Planning (KETEP) granted from the Ministry of Trade, Industry \& Energy, Republic of Korea (no. 20112010100100). Also this work was partially done during J. Chung's stay at Korea Electrotechnology Research Institute. J. Chung is grateful for support and kind hospitality.

\section{References}

[1] F. Andreu, J. M. Mazón, J. D. Rossi, and J. Toledo, "A nonlocal $p$-Laplacian evolution equation with Neumann boundary conditions," Journal de Mathématiques Pures et Appliquées, vol. 90, no. 2, pp. 201-227, 2008.

[2] L. A. Caffarelli, S. Salsa, and L. Silvestre, "Regularity estimates for the solution and the free boundary of the obstacle problem for the fractional Laplacian," Inventiones Mathematicae, vol. 171, no. 2, pp. 425-461, 2008.

[3] C. Carrillo and P. Fife, "Spatial effects in discrete generation population models," Journal of Mathematical Biology, vol. 50, no. 2, pp. 161-188, 2005.

[4] E. Chasseigne, M. Chaves, and J. D. Rossi, "Asymptotic behavior for nonlocal diffusion equations," Journal de Mathématiques Pures et Appliquées, vol. 86, no. 3, pp. 271-291, 2006.

[5] L. Silvestre, "Hölder estimates for solutions of integro differential equations like the fractional Laplace," Indiana University Mathematics Journal, vol. 55, no. 3, pp. 1155-1174, 2006.

[6] A. S. Ackleh and L. Ke, "Existence-uniqueness and long time behavior for a class of nonlocal nonlinear parabolic evolution equations," Proceedings of the American Mathematical Society, vol. 128, no. 12, pp. 3483-3492, 2000.

[7] M. Chipot and B. Lovat, "On the asymptotic behaviour of some nonlocal problems," Positivity, vol. 3, no. 1, pp. 65-81, 1999.

[8] M. Chipot and J. F. Rodrigues, "On a class of nonlocal nonlinear elliptic problems,” M2AN, vol. 26, no. 3, pp. 447-468, 1992.

[9] T. F. Ma and J. E. M. Rivera, "Positive solutions for a nonlinear nonlocal elliptic transmission problem," Applied Mathematics Letters, vol. 16, no. 2, pp. 243-248, 2003.

[10] L. Byszewski, "Theorems about the existence and uniqueness of solutions of a semilinear evolution nonlocal Cauchy problem," Journal of Mathematical Analysis and Applications, vol. 162, no. 2, pp. 494-505, 1991. 
[11] N. W. Ashcroft and N. D. Mermin, Solid State Physics, Brooks/ Cole, 1976.

[12] D. M. Rowe, Thermoelectrics Handbook, CRC Press, 2006.

[13] H. J. Goldsmid, Introduction to Thermoelectricity, Spring, 2010.

[14] G. J. Snyder and E. S. Toberer, "Complex thermoelectric materials," Nature Materials, vol. 7, no. 2, pp. 105-114, 2008.

[15] C. Hadjistassou, E. Kyriakides, and J. Georgiou, "Designing high efficiency segmented thermoelectric generators," Energy Conversion and Management, vol. 66, pp. 165-172, 2013.

[16] A. Figueroa and F. Vázquez, "Spectral and finite difference solutions of the hyperbolic heat transport equation for thermoelectric thin films," Applied Mathematics, vol. 4, no. 10, pp. 2227, 2013.

[17] G. J. Snyder and T. S. Ursell, "Thermoelectric efficiency and compatibility," Physical Review Letters, vol. 91, no. 14, Article ID 148301, 2003.

[18] M. W. Oh, J. H. Ahn, J. K. Lee et al., "Estimation of power generation from thermoelectric devices: model analysis and performance measurements," Electronic Materials Letters, vol. 6, no. 3, pp. 129-134, 2010.

[19] L. Onsager, "Reciprocal relations in irreversible processes. I," Physical Review, vol. 37, no. 4, pp. 405-426, 1931.

[20] L.-D. Zhao, S.-H. Lo, Y. Zhang et al., "Ultralow thermal conductivity and high thermoelectric figure of merit in SnSe crystals," Nature, vol. 508, no. 7496, pp. 373-377, 2014.

[21] P. B. Bailey, L. F. Shampine, and P. E. Waltman, Nonlinear Two Point Boundary Value Problems, Academic Press, 1968. 


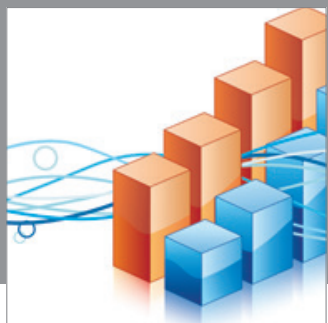

Advances in

Operations Research

mansans

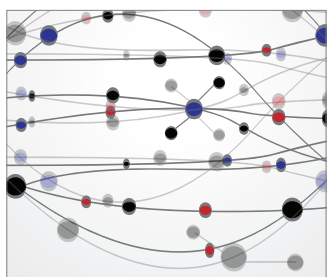

The Scientific World Journal
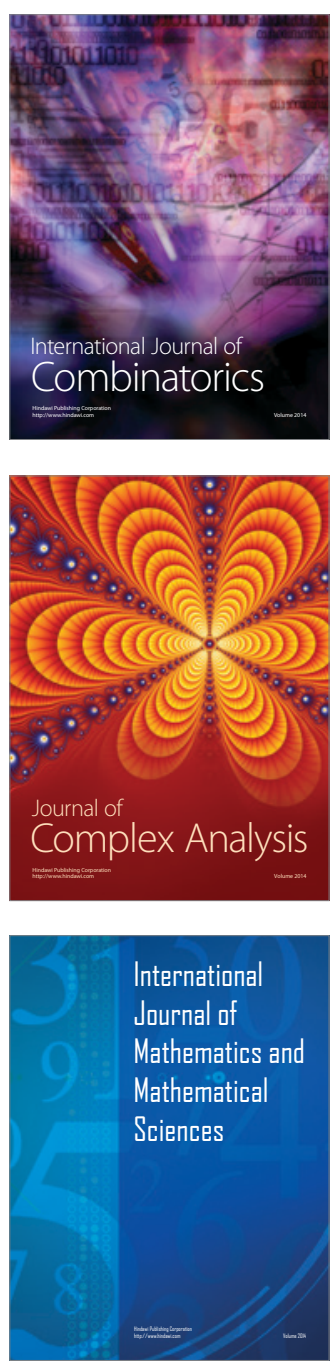
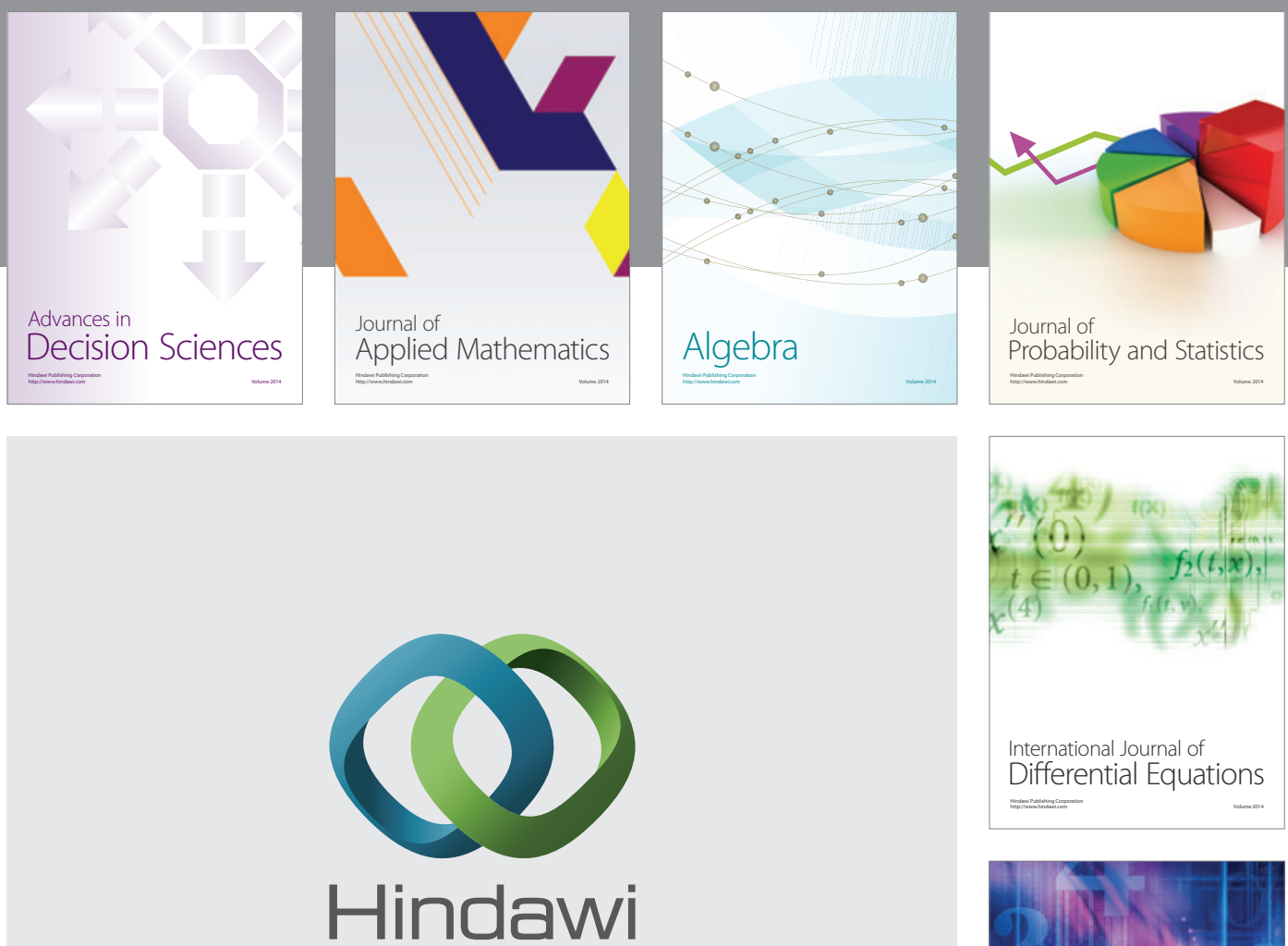

Submit your manuscripts at http://www.hindawi.com
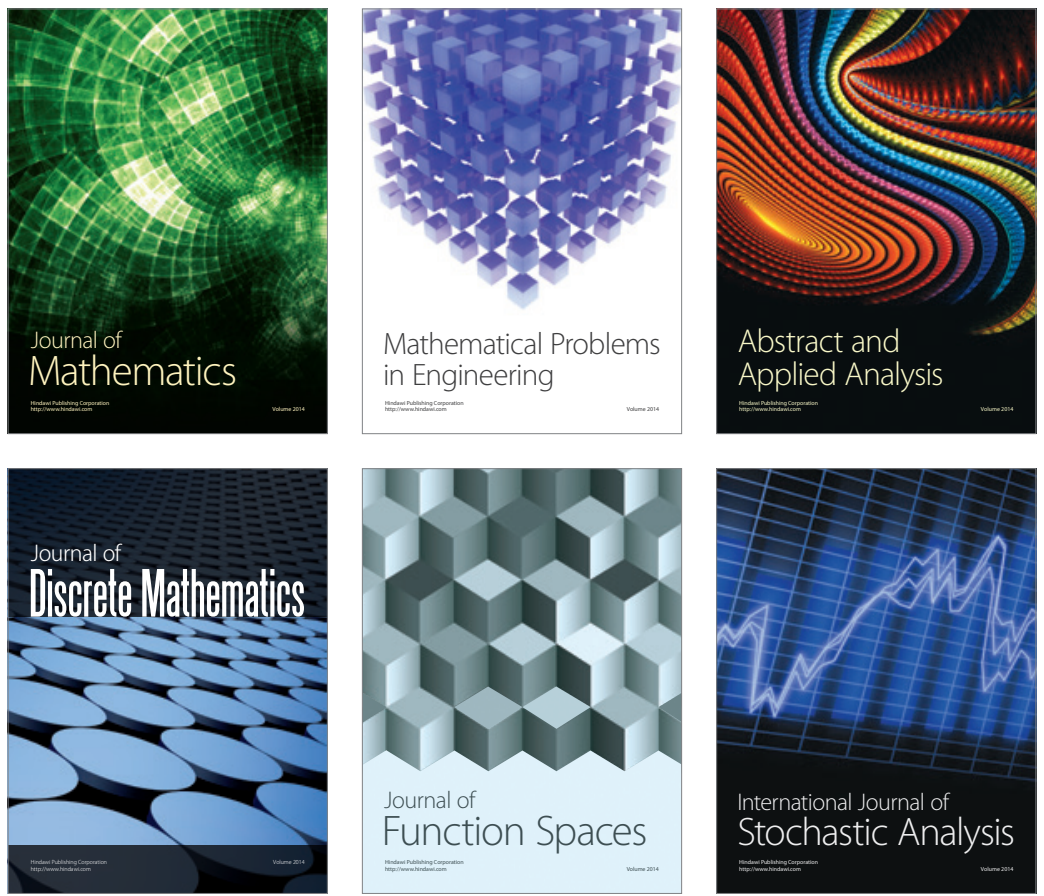

Journal of

Function Spaces

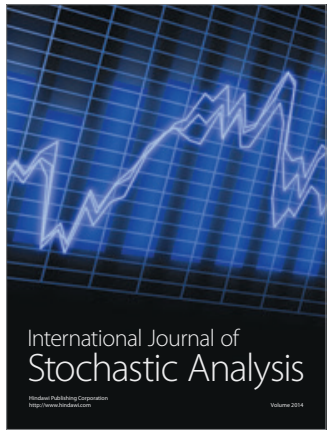

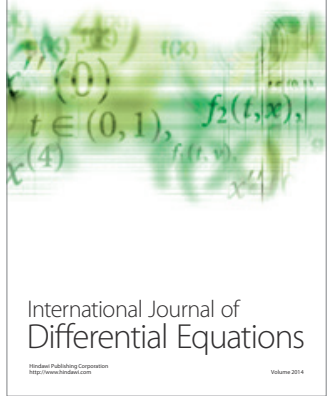
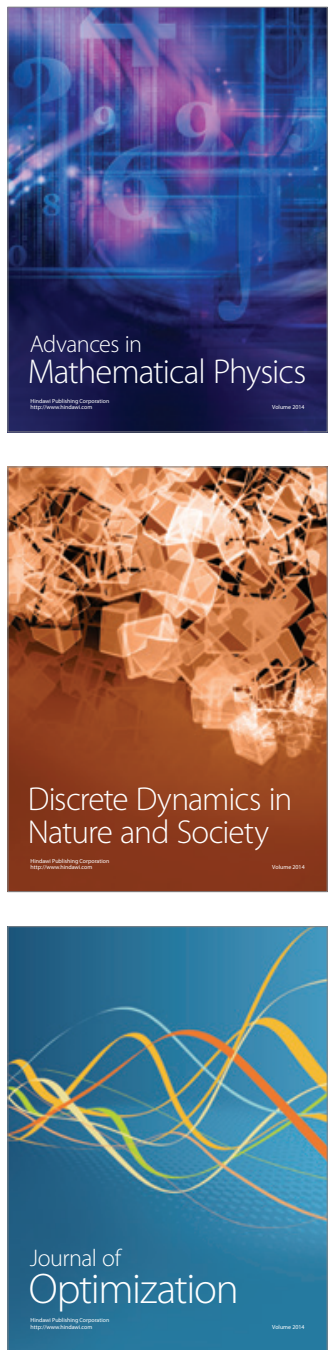